\title{
Deleterious reproductive effects of nilotinib in mouse model
}

\author{
Sinan Ozkavukcu@1,2, Nilay Kuscu ${ }^{3}$, Dileyra Adiguzel ${ }^{3}$, Guldane Cengiz-Seval ${ }^{4}$ and \\ Ciler Celik-Ozenci ${ }^{3}$ \\ ${ }^{1}$ Department of Histology and Embryology, Ankara University Faculty of Medicine, Ankara, Turkey, ${ }^{2}$ Center for \\ Assisted Reproduction, Department of Obstetrics and Gynecology, Ankara University Faculty of Medicine, Ankara, \\ Turkey, ${ }^{3}$ Department of Histology and Embryology, Akdeniz University Faculty of Medicine, Antalya, Turkey and \\ ${ }^{4}$ Department of Hematology, Ankara University Faculty of Medicine, Ankara, Turkey
}

Correspondence should be addressed to C Celik-Ozenci; Email: cilerozenci@akdeniz.edu.tr

\begin{abstract}
Nilotinib is a second-generation tyrosine kinase inhibitor (TKI) that is widely used to treat patients with Philadelphia chromosomepositive chronic myeloid leukaemia (CML). TKIs provided a significant improvement in terms of survival rates and disease-free period in CML; however, there is insufficient knowledge about their side effects, including reproductive toxicity. Since nearly half of the CML patients are in their reproductive age, and newly announced indications cover the treatment of the paediatric age groups, concerns arise about the effects of these drugs on the reproductive system, as there are no controlled preclinical studies. We investigated acute and long-term gonadotoxic and teratogenic effects of nilotinib, utilising a mouse model that simulates various clinical scenarios. We observed significant testicular damage in mice receiving nilotinib according to Johnsen's score analysis. Alterations were observed in female mice's number of follicles, as the primordial follicle numbers significantly decreased. Proliferating cell number in both genders' gonads decreased and apoptosis rate increased significantly. The nilotinib-received female and male mice's pregnancy rates were low compared to controls. A significant decrease in the thickness of the spongiotrophoblast and decidual layers of the placenta was detected in pregnancies consisting of male and/or female mice treated with nilotinib. The results of this study establish a critical point of view for clinical translation and indicate the importance of consulting patients for directing them to fertility preservation and contraception options for both genders before nilotinib treatment.

Reproduction (2021) 161 295-306
\end{abstract}

\section{Introduction}

Chronic myeloid leukaemia (CML) is a myeloproliferative disorder characterised by a chromosomal translocation between chromosomes 9 and $22(t(9 ; 22)(q 34 ; q 11))$, the Philadelphia chromosome, causing an abnormal gene fusion product; BCR-ABL1. This product acts as an active tyrosine kinase (TK), which stimulates cells for proliferation in a continuous manner, thus resulting in the clinical manifestations of CML. The prevalence of CML has been estimated to be 10-12/100,000 with a constant increase due to the dramatic improvement in these patients' survival. Today, with the use of tyrosine kinase inhibitors (TKIs), a near-normal life expectancy and a significant improvement in the quality of life can be achieved, but this can only be possible with the continuous use of the drug for years. Although the average age of CML diagnosis is in the 60s, this is 10-15 years earlier in Asian countries, and in general, 30-50\% of the patients can face this situation in their reproductive ages. After discovering the abnormal TK activity, efforts focused on the selective inhibition of TKs, and imatinib mesylate was the first chemical used in CML treatment.
Nilotinib, dasatinib, bosutinib, and ponatinib were developed and introduced following the first-generation TKIs, which have become the first-line treatment choice for CML (Kantarjian et al. 2003, Rosti et al. 2009).

Nilotinib is a second-generation $\mathrm{ABL}$ kinase inhibitor approved for CML therapy. It is structurally related to imatinib as it was created by replacing the $\mathrm{N}$-methylpiperazine binding group to optimise binding affinity and selectivity for Abl kinase. Thus, nilotinib became a selective inhibitor of $B C R-A B L$, unlike imatinib and dasatinib, and is 30 times more potent than imatinib. Nilotinib is actively prescribed as a first-line drug in patients with chronic phase and accelerated phase CML who have developed resistance to imatinib. In phase studies compared to imatinib, nilotinib achieved higher rates of complete cytogenetic and major molecular responses. Nilotinib has found intense clinical use as it is active against all BCR-ABL mutations, except for T315I (US Food and Drug Administration 2018). Recently, the FDA approved nilotinib for paediatric patients with newly diagnosed or resistant/intolerant Philadelphia chromosome-positive CML in the chronic phase (US Food and Drug Administration 2018). Despite its impact 
and future potential, and recently announced indications for the paediatric patient group, information on the gonadotoxic or teratogenic side effects of this drug is limited and depends on a few case reports involving only a small number of patients. In the literature, the adverse effects of nilotinib on reproduction have not been studied in detail. Normal births and miscarriage have been reported due to accidental pregnancies during drug use; however, the detrimental effects on gametes and gonads are unknown (Conchon et al. 2009, Berveiller et al. 2012, Alizadeh et al. 2015, Cheon et al. 2016).

It is well-known that TKs have essential roles in gametogenesis, fertilisation, and foetal development. The Src family of tyrosine protein kinases and c-kit are critical families of protein tyrosine kinases that act during spermatogenesis, Sertoli cell function, and Leydig cell development (Kierszenbaum 2006, Chen et al. 2009, Ijiri et al. 2012). It was shown that the first generation TKI, imatinib, impairs testicular growth and may cause infertility in offspring when used during pregnancy (Nurmio et al. 2007). Protein TK signalling also plays a vital role in oocyte maturation, meiosis, fertilisation, and zygote development (Mcginnis et al. 2011).

Although contraception is recommended during TKI treatment, pregnancies occur under drug use, leaving healthcare providers in a conflicting position (Barkoulas \& Hall 2018). Since there is no comprehensive data on nilotinib's reproductive and foetal toxicity, patients may not be given adequate consultation on fertility preservation options (Palani et al. 2015).

This study aimed to evaluate the acute and long-term gonadotoxicity and teratogenicity of nilotinib and to assess the fertility of the first-generation offspring born from males and females who received nilotinib. The hypothetic wash-out period of nilotinib was also tested by following the reproductive parameters of the mice from both genders after drug cessation.

\section{Materials and methods}

Five-weeks old, 20-22 g, Balb/c breed female and male mice were used for the study, obtained from the Akdeniz University Animal Research Unit, Antalya, Turkey, and the experimental protocol was approved by the Animal Care and Use Committee of Akdeniz University, Faculty of Medicine (decision date 03.10.2016 and number 76). The weights of all mice were recorded, and standard care rules were followed. They were kept alive in standard cages and were fed with pellet-type fabricated food; containing $21 \%$ crude protein specially designed for small experimental animals and were given daily in the drinking water. Appropriate laboratory conditions $\left(22 \pm 10^{\circ} \mathrm{C}, 12 \mathrm{~h}\right.$ light: $12 \mathrm{~h}$ darkness (07:00 $\mathrm{h} / 19: 00 \mathrm{~h}$ ) cycle) were provided during the experiment period. Female mice were kept two mice per cage, while male mice were kept alone. In mating groups, one male mouse was distributed per cage to mate with two female mice.

The water-soluble form of nilotinib (Tasigna, Novartis Pharmaceuticals Corporation, Basel, Switzerland) was supplemented in the animals' drinking water daily in the morning, making sure that it was completely dissolved at the concentration of $20 \mathrm{mg} / \mathrm{kg}$ per mouse (Jensen et al. 2006). The content was carefully monitored so that the animals entirely consumed the water. Experimental groups and their controls with the tested outcome parameters are summarised in Fig. 1.

\section{Experimental design on the acute and chronic gonadotoxicity $(G)$ of nilotinib}

To detect gonadotoxic effects, male and female mice received nilotinib for 2 months (G-NM, nilotinib male and G-NF, nilotinib female, $n=4$ for each) and their controls (G-CM and G-CF, $n=4$ for each) received only drinking water. Sixteen mice were then sacrificed by cervical dislocation, and their testicular $(n=16)$ and ovarian tissues $(n=16)$ were fixed in Bouin's solution. Additionally, the weight of the vital organs, lungs, kidneys, and livers of those animals was noted.

To reveal if the gonadotoxic effects were reversible, we designed a post-nilotinib (G-PNM and G-PNF, $n=4$ for each) group to simulate individuals who wanted to conceive after receiving a nilotinib treatment. In this group, after 2 months of nilotinib exposure, the follow up of the mice went on without receiving nilotinib for another 35 days in males, considering the duration of the spermatogenic cycle, and another 15 days in females, which means at least three oestrus cycles in mice. For this group's control, to equalise the animals' reproductive ages, male (G-CPNM, $n=4$ ) and female (G-CPNF, $n=4$ ) control post-nilotinib groups were recruited where the mice received only drinking water for 95 days for males and 75 days for females.

For the determination of the gonadotoxic effects in nilotinib and post-nilotinib groups, the following parameters were assessed and compared within groups including controls, after the designated experimental periods: bodyweight of the mice, testicular and ovarian weights, weights of the vital organs, immunohistochemical determination of testicular VASA for germ cells, apoptosis by cleaved caspase-3 (CC3) and proliferation by the proliferating cell nuclear antigen (PCNA), Johnsen's scoring for the evaluation of spermatogenesis, and primordial follicle count for the ovarian reserve using hematoxylin-eosin staining.

\section{Experimental design on the teratogenicity $(T)$ of nilotinib}

For 2 months, nilotinib was added to the drinking water of male (NM) and female (NF) mice at a dose of $20 \mathrm{mg} / \mathrm{kg} /$ day per mouse. Then the following cross-mating groups were created along with two female oestrus cycles with the participation of control males (CM) and females (CF):

1. T-NMCF: The group in which the male mice that received nilotinib $(n=2)$ were mated with the control females ( $n$ $=4$ ). This group aimed to simulate the obstetric outcomes when only the male partner received nilotinib before pregnancy and to demonstrate the alterations in male reproductive function.

2. T-CMNF: The group in which control males $(n=4)$ and nilotinib-received females ( $n=8,4$ in two subgroups) mated. 
Nilotinib Gonadotoxicity Experiments

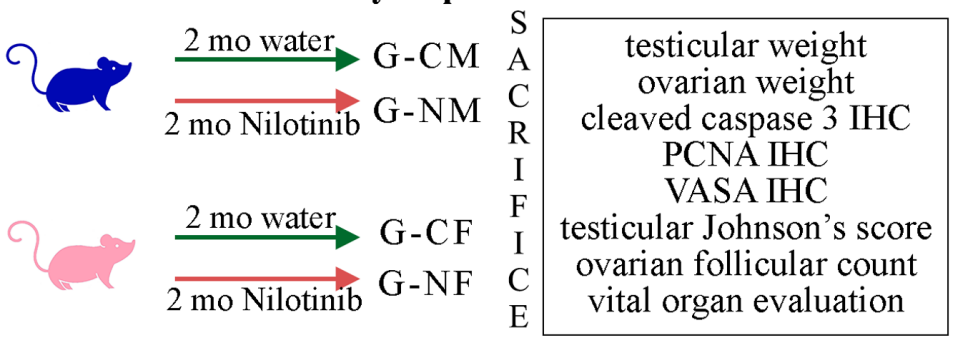

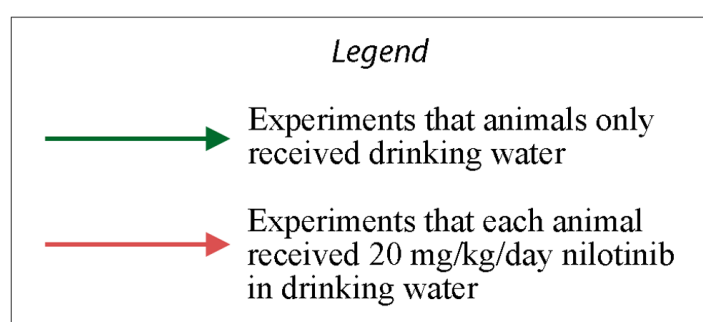

\section{Post-Nilotinib Gonadotoxicity Experiments}

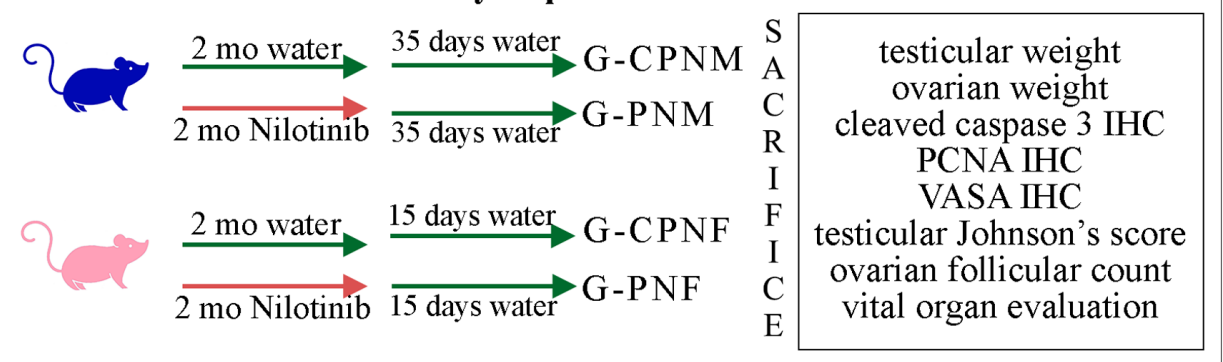

G: Gonadotoxicity

C: Control

M: $\quad$ Male

F: Female

N: Nilotinib

PN: Post-Nilotinib

T: Teratogenicity

Term: Nilotinib terminated upon pregnancy

Cont: Nilotinib continued during pregnancy

\section{Nilotinib Teratogenicity Experiments}

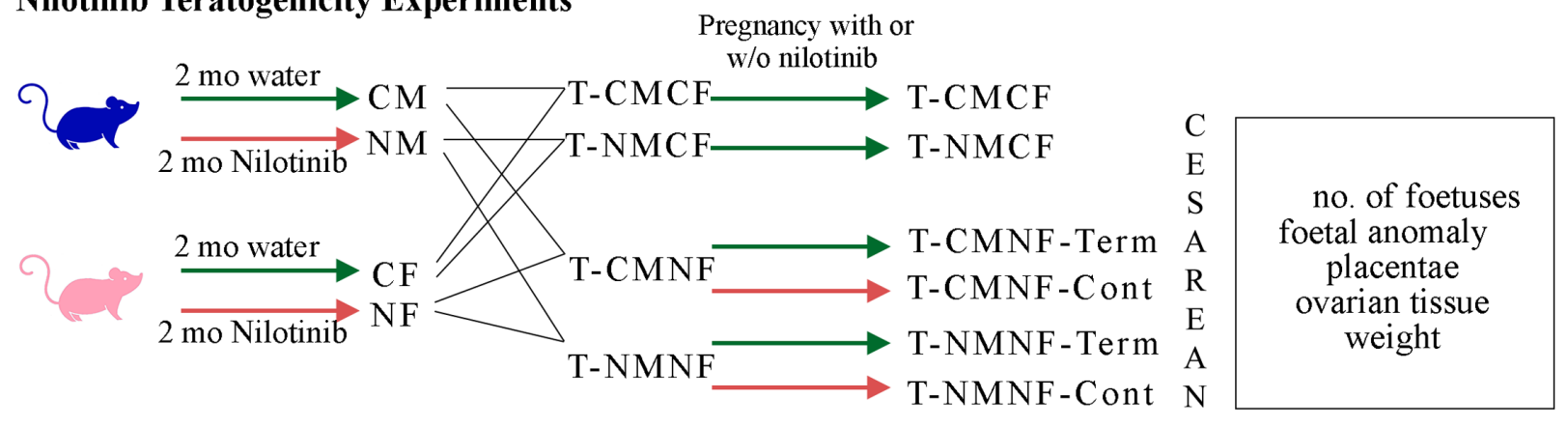

Fertility of the Offspring Experiments

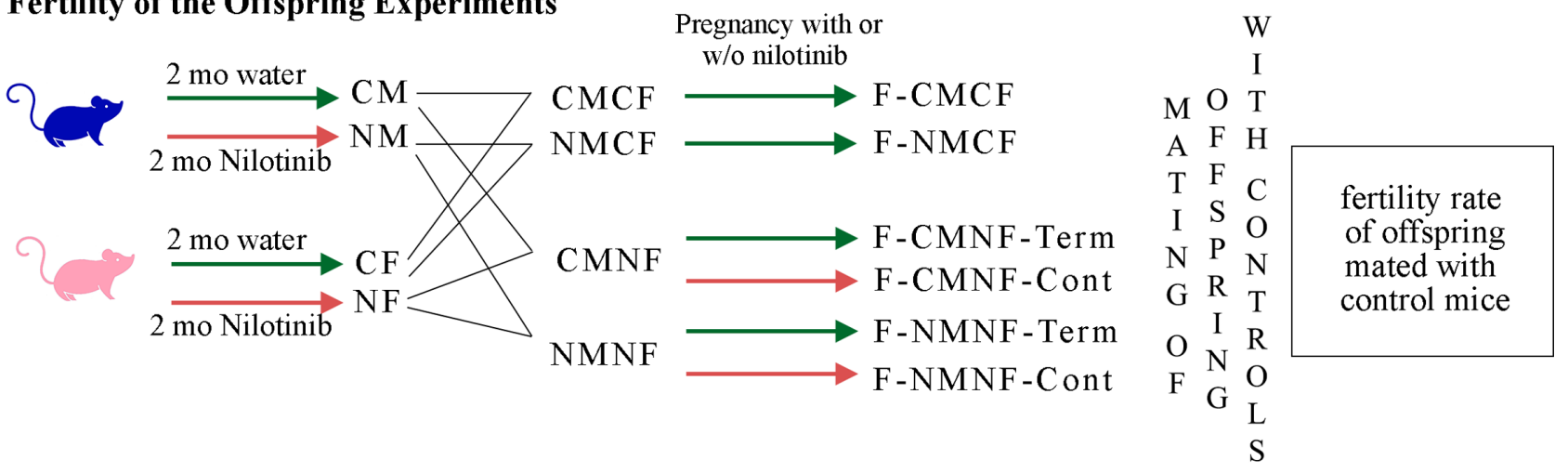

Figure 1 Summary of the experimental model and parameters evaluated in experimental groups. IHC, immunohistochemistry; PCNA, proliferating cell nuclear antigen; mo, month; w/o, without; no,: number.

This group aimed to simulate the obstetric outcomes when only the female partner received nilotinib before pregnancy. Moreover, two additional subgroups were also created. The first in which the nilotinib treatment was immediately terminated (T-CMNF-Term, $n=4$ ) upon pregnancy onset, and the second in which nilotinib treatment was continued despite pregnancy until term (T-CMNF-Cont, $n=4$ ).

3. T-NMNF: The group in which nilotinib-received males $(n=4)$ mated nilotinib-received females $(n=8,4$ in two subgroups). This group aimed to test the obstetric outcomes 
of an extreme condition when both partners received nilotinib before pregnancy. As in the previous group, this group also consisted of two subgroups. The first in which the nilotinib treatment was immediately terminated (T- NMNF-Term, $n=4$ ) upon pregnancy onset, and the second in which nilotinib treatment was continued despite pregnancy until term (T- NMNF-Cont, $n=4$ ).

4. T-CMCF: The control group in which mated male $(n=2)$ and female $(n=4)$ mice were only treated with drinking water without nilotinib.

Fecundation was checked by investigating the vaginal plaque after the mating period. On the 20th day of pregnancy, the weights of all female mice were recorded, and delivery by caesarean section was performed. Ovarian tissues, foetuses, placentae, and their attachments from mice after caesarean section were placed in $10 \%$ buffered formalin solution. The number of pregnancies, animal weight, foetus number, offspring weight, and placentas were evaluated together with their morphological features in all groups. Additionally, body weight, ovarian weight, ovarian apoptosis and proliferation (according to cleaved caspase - 3 (CC3) and proliferating cell nuclear antigen (PCNA) immunohistochemistry), primordial follicle count (hematoxylin-eosin staining) was carried out.

\section{Histological analysis of placentae}

Histological analysis was undertaken to evaluate nilotinib's effect on the mature placenta. Placental tissues were fixed in $10 \%$ buffered formalin for $24 \mathrm{~h}$ for histological assessment. The samples were processed for paraffin sectioning and serially sectioned at $5 \mu \mathrm{m}$ thickness for almost 100 slides for each placenta to reach the central level to allow all the placenta zones to be measured accurately. Measurements including the area of the labyrinth zone, the spongiotrophoblast zone, and the decidual zone were taken and compared between the treatment groups ( $n=3$ placentas per group, $n=3$ animals per group).

\section{Experimental design on the fertility of the parents and the offspring $(F)$}

Additional exposure and control mating groups were created under the same scenario with the teratogenicity groups; thus, the following subgroups were formed: F-CMCF, F-NMCF, F-CMNF-Term, F-CMNF-Cont, F- NMNF-Term, and F- NMNFCont. The pregnancy of four females from each group was followed to term, and delivery was achieved. When the offspring reached adulthood at week 5, they were mated with same-aged control mice (female or male), which did not receive nilotinib. Studies in this experimental group were terminated following the determination of the fertility status of the first-generation offspring and the number of deliveries were compared. Additionally, in the experimental groups of CMCF, NMCF, CMNF-Term, and NMNF-Term, the pregnancy rate (PR) and the live birth rate (LBR) for each group were calculated with the formulation used below:

\footnotetext{
$\mathrm{PR}=\frac{\text { the number of pregnant mice in the group }}{\text { all the female mice designated to the group for mating }} \times 100$
}

$$
\mathrm{LBR}=\frac{\text { the number of mice that naturally gave }}{\text { birth to live pups in the group }} \begin{aligned}
& \text { (all the female mice designated to the group for } \\
& \text { mating }- \text { the pregnancies ended with a caesarean) }
\end{aligned} \times 100
$$

\section{Histologic evaluation of seminiferous epithelium (Johnsen's scoring)}

The maturity of the germinal epithelium was staged using the modified Johnsen's testicular biopsy score (Johnsen 1970). Using $\times 400$ magnification under the light microscope, 100 seminiferous tubules were evaluated in each tissue section. A score between 1 and 10 was given to each tubule, and the average score of 100 tubules was recorded as the Johnsen's score.

\section{Histological analysis and follicle count in the ovaries}

Ovaries were fixed in Bouin's fixative and embedded in paraffin. Five micrometre-thick sections were taken from paraffin blocks and stained with hematoxylin-eosin. Each fifth consecutive section was taken for follicle evaluation, thereby preventing duplicate counts of the same follicle, and the histologic assessment of the ovarian follicles was performed (Ross \& Pawlina 2016).

\section{Immunohistochemistry}

Testicular and ovarian tissues, fixed in 5\% (v/v) formaldehyde (Merck, Sigma-Aldrich GmbH Taufkirchen, Germany; cat no: F8775) for $24 \mathrm{~h}$, were dehydrated through a graded ethanol series and embedded in paraffin. Five micrometre-cut sections were taken. After deparaffinisation and rehydration, citrate buffer ( $\mathrm{pH}$ 6.0) was used for antigen retrieval using a microwave. Subsequently, the slides were washed in PBS, and endogenous peroxidase activity was blocked by $3 \%$ hydrogen peroxide in methanol for $15 \mathrm{~min}$ at room temperature. After washing with PBS, the sections were treated with an ultra- $V$ block (Thermo; cat no: TA-125-UB) and incubated with primary antibody rabbit anti-VASA (1:200 dilution; Abcam, cat no: ab13840); mouse anti-PCNA (1:1000 dilution; Dako; cat no: PC10) or rabbit anti-CC3 (1:500 dilution; Cell Signaling; cat no: $9661 \mathrm{~S})$ overnight at $4{ }^{\circ} \mathrm{C}$. Next day, after washing out the primary antibody, slides were incubated for $60 \mathrm{~min}$ at room temperature with anti-rabbit (Vector, USA; BA-1000, 1/500) and anti-mouse secondary antibodies (Vecto; BA-2000, $1 / 500$ ) followed by incubation with horseradish peroxidaseconjugated streptavidin (Thermo Scientific; TS-125-HR) for $30 \mathrm{~min}$ at room temperature. All incubation steps were performed in a humidified chamber to avoid dehydration of the slides. Positive immunoreactions were visualised with diaminobenzidine (DAB)-peroxidase substrate (Sigma; cat no: D4168), and counterstaining was performed with hematoxylin. Pictures were taken with Spot Advanced Imaging Software (Diagnostic Instruments, Inc., Sterling Heights, MI, USA). Expression levels of VASA, PCNA, and CC3 in testicular and ovarian tissues were calculated using ImageJ software $(\mathrm{NIH})$. The percentage of stained cell intensity was measured and then divided by total area. 


\section{Statistical evaluation}

Data were reported as mean \pm S.E.M.. For the weight ratios of the vital organs and the gonads, unpaired $t$-test and for the body weight and ovarian follicle count, multiple $t$-tests were used to describe the differences among the groups for parametric data. Group comparisons were performed with one-way ANOVA test for parametric data or Kruskal-Wallis one-way ANOVA on ranks test for non-parametric data followed by Tukey's post hoc test for foetus number per mother, foetus/placenta weight ratio, and pup weight to describe any differences between the groups. Chi-square test was performed for the pregnancy and live birth rates. All statistical tests were performed using Sigma Stat 3.5 software (Jandel Scientific Corp.) and $P<0.05$ was considered statistically significant.

\section{Results}

\section{Extragonadal effects of nilotinib}

Even though the toxicity of nilotinib on vital organs was not the primary goal of the current study, the extragonadal organs, kidney, liver, lung, and heart were weighed and proportioned to the bodyweight to reveal whether the overall body system was affected, to outline a robust conclusion on the reproductive system effects. According to findings obtained from the gonadotoxicity group, it was noted that nilotinib application did not generate any significant extragonadal changes by means of organ weight when compared to the control group.

\section{Gonadotoxicity (G) of nilotinib on the male reproductive system}

Findings in the male mice group with body weight, testis/body weight ratio, histological evaluations with hematoxylin-eosin staining, the results of Johnsen's scoring, and expression of PCNA, VASA, and CC3 are summarised in Fig. 2. Nilotinib treatment caused a certain decrease in animal weight, although the difference was not statistically significant during the first four weeks. Starting from the fifth week, the bodyweight of the male mice decreased significantly in the nilotinib group (Fig. 2A). However, the testis/body weight ratio was not found to be different between the groups (control: $0.002627 \pm 0.0002881$ nilotinib: $0.003095 \pm 0.0004204, P=0.3486$ ) (Fig. 2B). When histological sections were evaluated, the tubules were damaged, and the cellular structures were disorganised, signifying a remarkable testicular deterioration in mice treated with nilotinib. Specifically, unlike the controls, gaps in the intertubular areas and disorganisation in the seminiferous epithelium hierarchy were detected in the nilotinib group. It was noticed that the borders between the adluminal and basal compartments were lost, and there were large vacuole-like spaces within the epithelium. Normal-looking seminiferous tubules
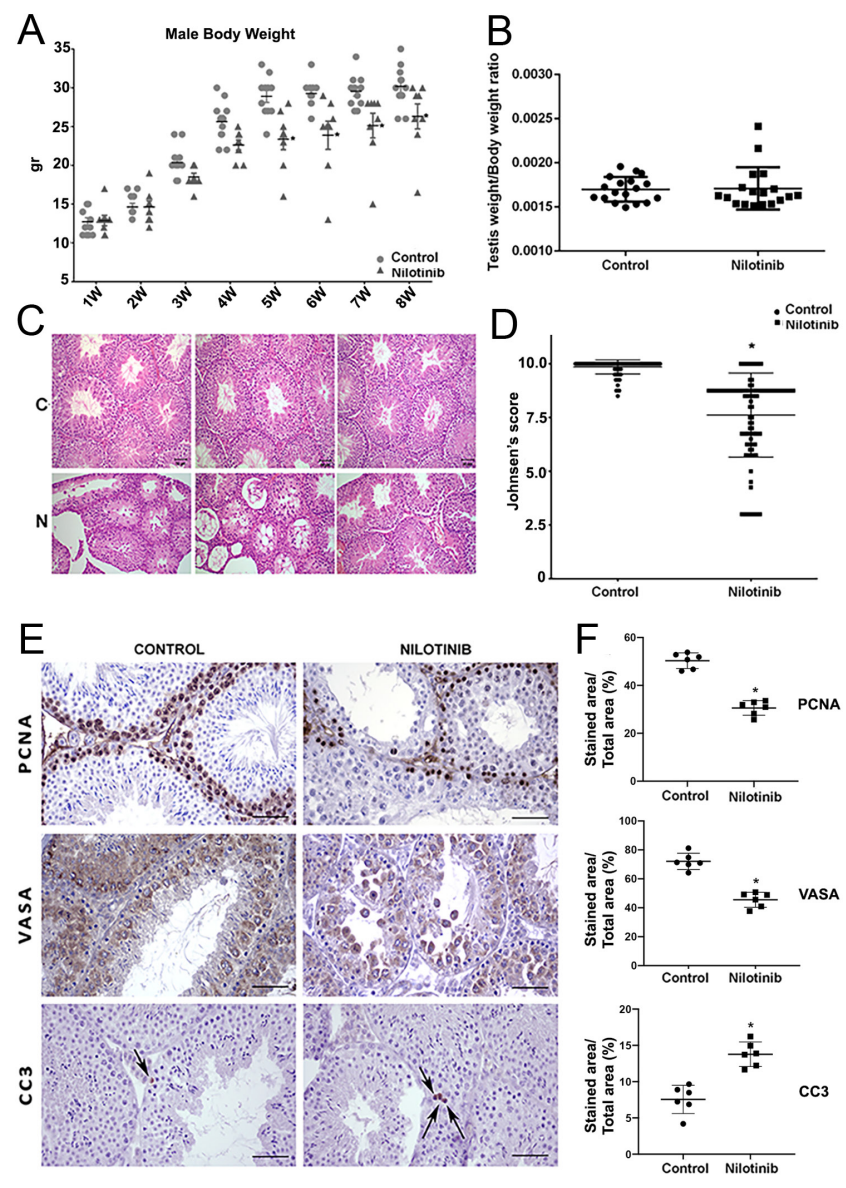

Figure 2 The findings of the gonadotoxicity experiments in male mice. (A) Bodyweight change vs time graph, (B) testis/body weight ratio, (C) histological evaluations with hematoxylin-eosin staining, (D) the results of Johnsen's scoring, (E) PCNA, VASA, and CC3 immunohistochemistry staining (arrows indicate apoptotic cells), and (F) ImageJ evaluation results. Scale bars indicate $50 \mu \mathrm{m}$.

were also present. In the pathological tubules, the basal membrane thickening was not observed (Fig. 2C). The mean Johnsen's score in testes of male mice that received nilotinib was $7.62 \pm 1.95$ vs $9.85 \pm 0.33$ in the control group, where the difference between the two groups was statistically significant $(P<0.0001)$ (Fig. 2D). When the immunohistochemistry results were evaluated, PCNA expression revealed a significant decrease in the nilotinib group compared to the control group $(30.58 \pm 3.03$ vs $50.34 \pm 3.24$, respectively, $P<0.0001)$. In contrast, CC3 expression increased significantly $(13.78 \pm 1.69$ and $7.56 \pm 1.95$, respectively, $P<0.0001)$. Although the intensity of VASA expression was similar in both groups, it was found that germ cells expressing VASA in the nilotinib group displayed a remarkable disorganisation pattern in the seminiferous tubules, as the germs cells were scattered within the seminiferous epithelium, destroying the compartmental organisation of the spermatogenic epithelium (Fig. 2E). Due to irregular 
cell placement, VASA expression, calculated in the nilotinib group, was found to be significantly decreased compared to the controls (45.52 \pm 5.19 vs $72.10 \pm 5.64$, respectively, $P<0.0001$ ) (Fig. $2 \mathrm{~F}$ ).

\section{Long-term effects of nilotinib on the male reproductive system}

After 2 months of nilotinib exposure via drinking water at a dose of $20 \mathrm{mg} / \mathrm{kg} /$ day per mouse, male mice were followed for another 35 days (considering the duration of the spermatogenic cycle in the mouse), receiving drinking water without nilotinib supplementation. This experiment aimed to simulate whether the effects of nilotinib on patients' gonads were reversible (PN-post-nilotinib group). PCNA, VASA, and CC3 immunostaining, testis/bodyweight ratio, histological evaluations, and the results of Johnsen's scoring after hematoxylin-eosin staining of the post-nilotinib group are summarised in Fig. 3. It was revealed that the difference between testis/bodyweight ratio between groups was comparable (control: $3.32 \pm 0.13$, postnilotinib: $3.05 \pm 0.24, P=0.3606)$. When histological sections were evaluated, the tubules were detected to be damaged and were disorganised in the testicles of the PN group (Fig. 3B); thus, a significant difference was observed when Johnsen's scoring was evaluated in the $\mathrm{PN}$ group when compared to the control $(7.43 \pm 1.84$ vs $9.77 \pm 0.23$, respectively, $P<0.0001$ ) (Fig. 3C). Immunohistochemistry results showed that PCNA expression in the PN group decreased significantly when compared to the controls $(35.89 \pm 4.07$ vs $48.32 \pm 3.73$, respectively, $P=0.0003$ ) and CC3 expression increased significantly $(20.00 \pm 0.86$ vs $9.96 \pm 1.24$, respectively, $P<0.0001)$. VASA expression intensity was determined to be similar in both groups (control: $71.63 \pm 2.78, \mathrm{PN}$ : $71.46 \pm 5.82, P=0.9505$ ) (Fig. 3E). Despite a similarity in VASA expression, it was noteworthy that spermatogenic cells' topography in the seminiferous tubules showed irregularity. The displacement of the cells towards the luminal compartment was noted (Fig. 3D).

\section{Gonadotoxicity $(G)$ of nilotinib on the female reproductive system}

Findings in the female mice group with bodyweight, ovary/bodyweight ratio, histological evaluations with hematoxylin-eosin staining, and the results of follicle count, PCNA, and CC3 results are summarised in Fig. 4. Similar to the male group, nilotinib caused a notable decrease in animal weight, although the difference was not statistically significant during the first four weeks. There was no significant difference in the ovary/ bodyweight ratio in the nilotinib group compared to controls $(0.30 \pm 0.02$ and $0.23 \pm 0.04, P=0.2251)$ (Fig. 4B). When histological sections were evaluated,
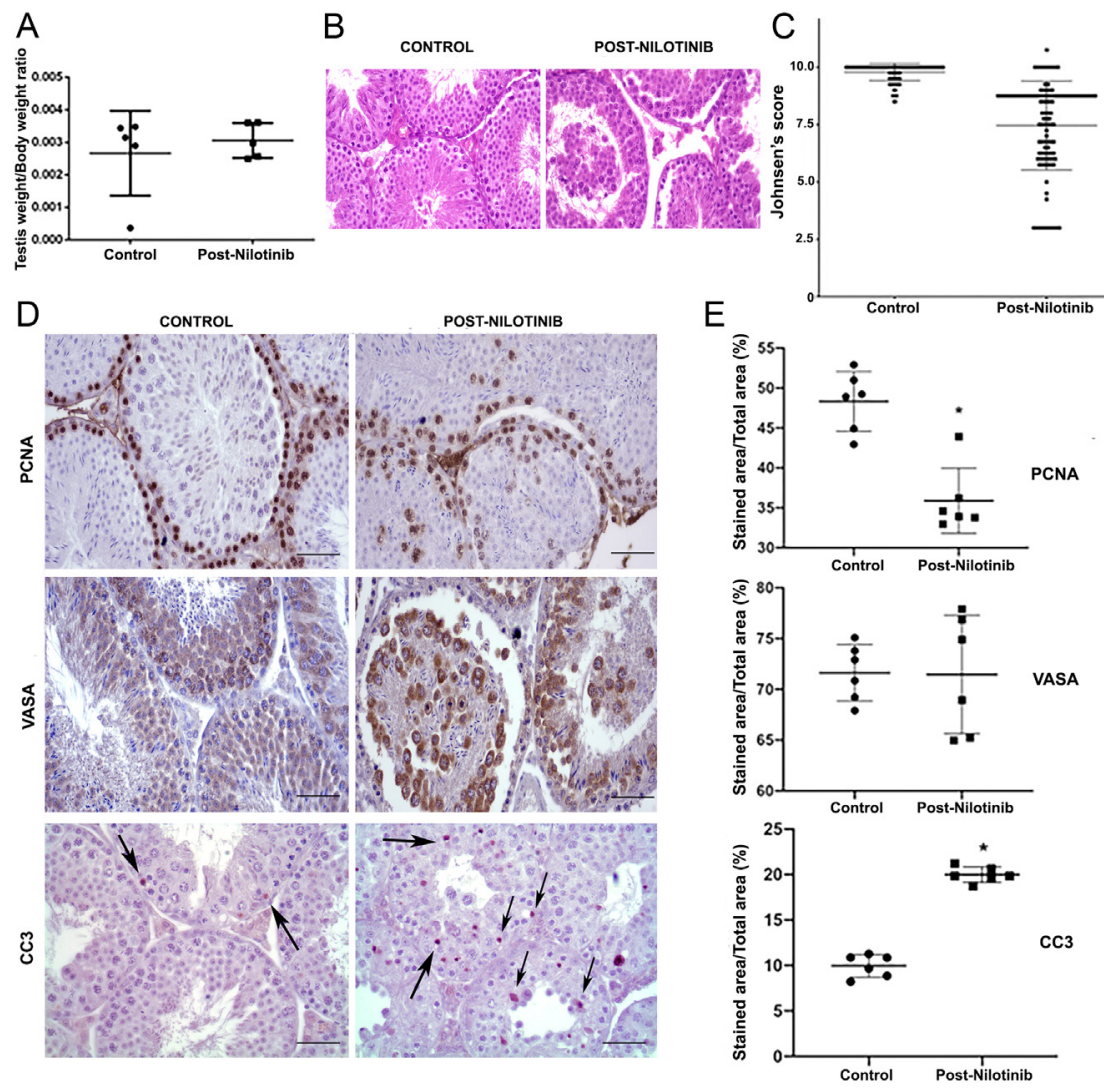

Figure 3 The findings of the post-nilotinib group in male mice. (A) Testis/bodyweight ratio, (B) histological evaluations with hematoxylin-eosin staining, $(C)$ the results of Johnsen's scoring, (D) PCNA, VASA, and CC3 immunohistochemistry (arrows indicate apoptotic cells), and (E) ImageJ evaluation results. Scale bars indicate $50 \mu \mathrm{m}$. 

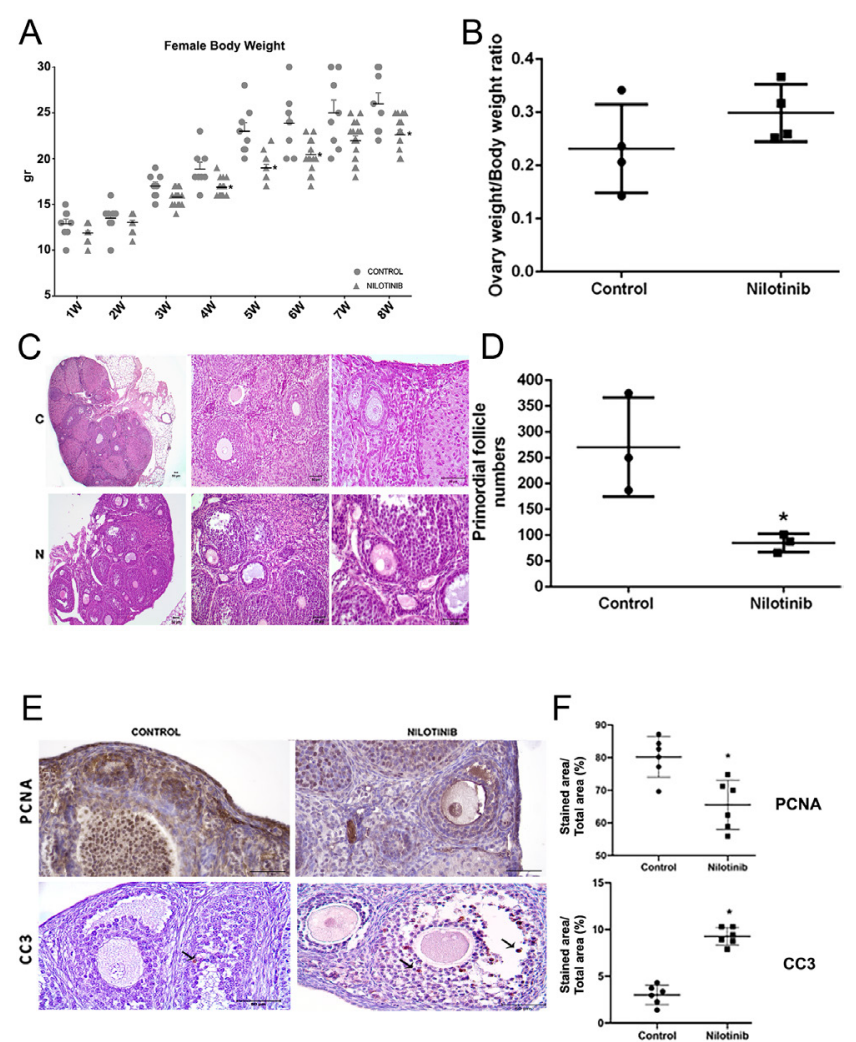

Figure 4 The findings of the gonadotoxicity experiments in female mice. (A) Body weight change graph, (B) ovary/bodyweight ratio, (C) histological evaluations with hematoxylin-eosin staining, (D) the results of primordial follicle count, (E) PCNA and CC3

immunohistochemistry staining (arrows indicate apoptotic cells), and (F) ImageJ evaluation results. Scale bars indicate $50 \mu \mathrm{m}$.

it was found that the nilotinib treatment caused irregularities in follicle structure and vacuolisation in the oocytes (Fig. 4C). Accordingly, when the follicles were counted, a significant decrease in the number of primordial follicles in the nilotinib group was observed when compared to the control group $(P<0.0001)$ (Fig. 4D). When immunohistochemistry results were evaluated, PCNA expression decreased significantly $(65.56 \pm 7.53$ vs $80.21 \pm 6.20$, respectively, $P=0.0043)$, and CC3 expression increased significantly $(9.27 \pm 0.94$ and $3.00 \pm 1.05$, respectively, $P<0.0001$ ) in the nilotinib group when compared to the control group (Fig. 4E and F).

\section{Long-term effects of nilotinib on the female reproductive system}

Drinking water of female mice was supplemented with nilotinib at a dose of $20 \mathrm{mg} / \mathrm{kg} /$ day per mouse for 2 months. Later, for an additional 15 days (three oestrus cycles), the mice were followed without any drug exposure. This group aimed to simulate whether the reproductive effects of nilotinib were reversible in females. The ovary weight/ bodyweight ratio, histological evaluations, the follicle count results by hematoxylin-eosin staining, and PCNA and CC3 results determined by immunohistochemistry are summarised in Fig. 5. The ratio of ovary weight/body weight was comparable between the two groups (control: $0.20 \pm 0.01$ vs post-nilotinib: $0.19 \pm 0.01, P=0.5267$ ) When histological sections were examined, it was evident that the ovarian structure was morphologically damaged in the $\mathrm{PN}$ group when compared to the controls. However, when the follicle count was evaluated, there was no significant difference between the PN group and the controls. Immunohistochemistry results revealed that PCNA expression decreased significantly in the $\mathrm{PN}$ group $(79.61 \pm 2.92$ vs $72.64 \pm 2.59$, respectively, $P=0.0014)$, and CC3 expression increased significantly when compared to controls $(1.98 \pm 0.31$ and $9.03 \pm 1.73$, respectively, $P<0.0001)$.

\section{Teratogenicity $(T)$ of nilotinib}

In order to evaluate the foetus and placenta weights, females in all groups were sacrificed on the 20th day of pregnancy after vaginal plug detection, and their foetuses and placentas were obtained by caesarean section. The number of foetuses per mother (Fig. 6A) and the ratio of foetal weight to placental weight were compared between all groups. While the number of foetuses per mother did not differ significantly between the groups, it was remarkable that there was a variation in the number of foetuses per mother, especially when nilotinib was administered to any of the parents. Furthermore, this variation was even more remarkable when nilotinib was administered to both parents. On the other hand, the foetal weight/placental weight ratio decreased in the groups in which both parents received nilotinib, and this decrease was significant in the T-NMNF-Term group $(P<0.05)$ (Fig. $6 \mathrm{~B})$. When the morphology of the foetuses was examined, no major malformation was found (Fig. 6C). There was no significant difference between the groups regarding the first-generation offspring's weight (Fig. 6D).

To examine the possible effects of nilotinib on the placenta in more detail, histological analysis of the mature placentas obtained on the 20th day of pregnancy and the area measurements of the compartments of these placentas were performed. Females in CF-NM, NF-CMTerm, NF-CM-Cont., NF-NM-Term, NF-NM-Cont. groups had smaller placentae than the control group (Fig. 7A). Within each placental section, the area of the labyrinth, spongiotrophoblast (junctional zone), and the decidual zone were measured (Fig. 7B, C and D). Exposure of any parent to nilotinib significantly reduced the area of the spongiotrophoblast zone $(P<0.05)$ and the decidual zone $(P<0.05)$ (Fig. 7A, B, C and D). In the groups where both parents received nilotinib, the decrease in the spongiotrophoblast zone was even more pronounced. 


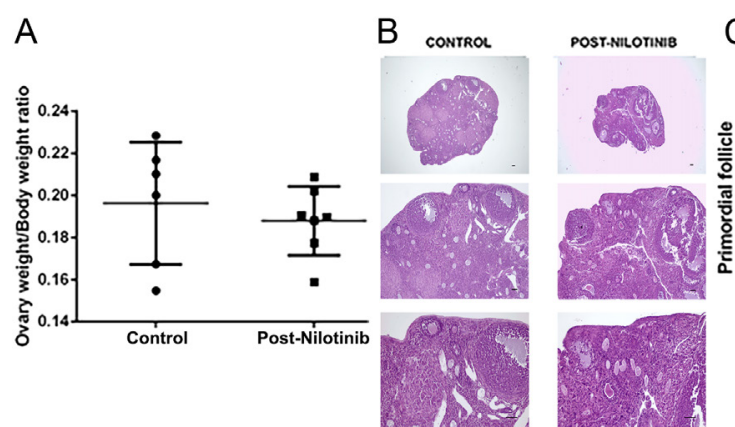

D

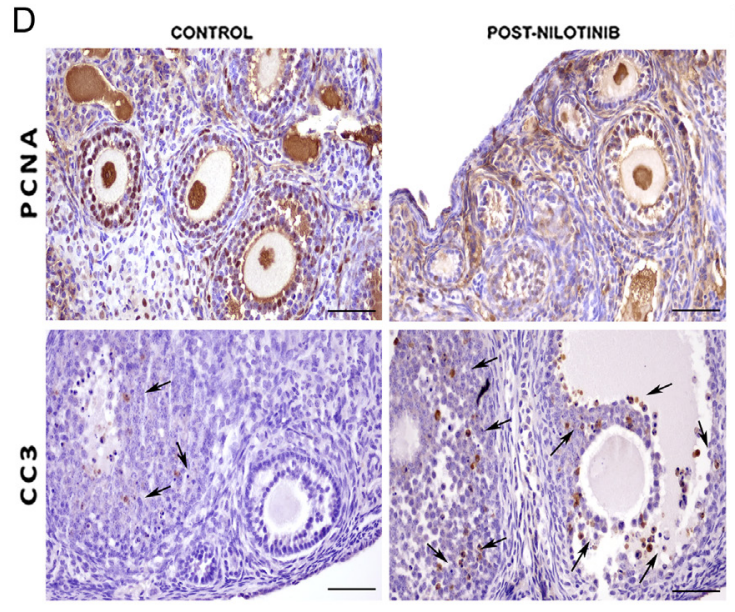

C

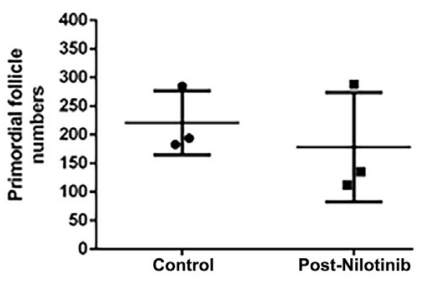

E
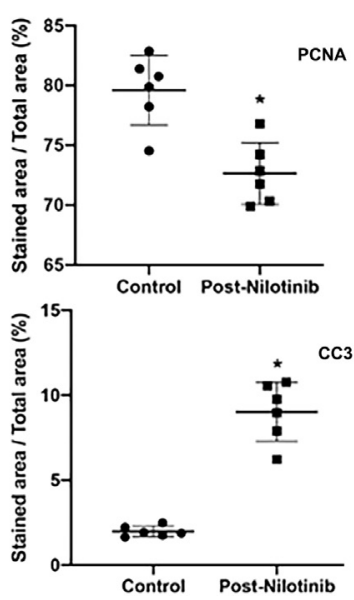

Figure 5 The findings of the post-nilotinib group in female mice. (A) Ovary weight/ bodyweight ratio, (B) histological evaluations with hematoxylin-eosin staining, (C) the results of primordial follicle count, (D) PCNA and CC3 immunohistochemistry (arrows indicate apoptotic cells), and (E) ImageJ evaluation results. Scale bars indicate $50 \mu \mathrm{m}$.

\section{Effect of nilotinib on the fertility of the parents and offspring $(F)$}

The nilotinib-received and control adults' pregnancy and live birth rates of the parents were summarised in Fig. 8. Pregnancy rate in CMCF group was calculated in 14 females and 11 pregnancies occurred $(78.6 \%)$, in NMCF group was calculated in 12 females and 7 pregnancies
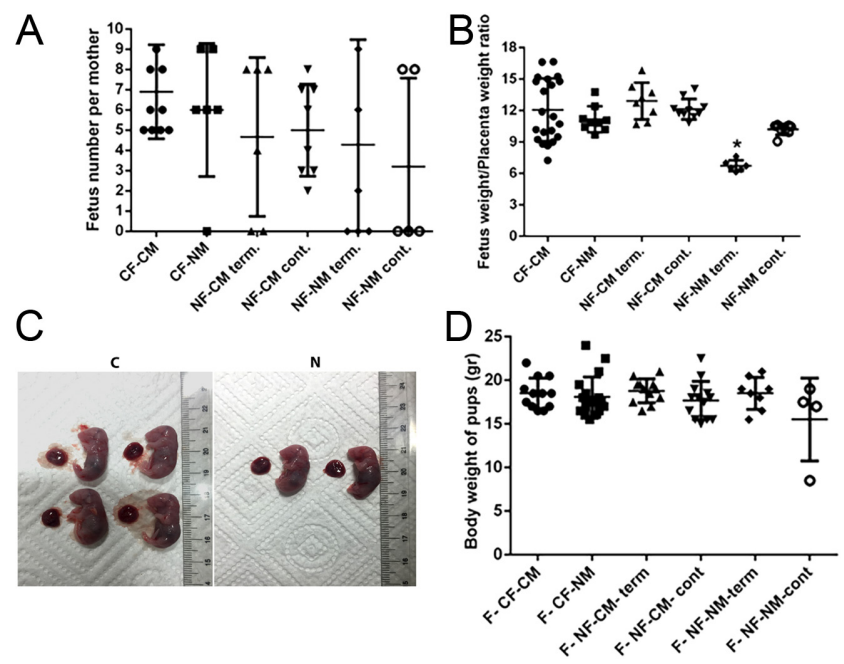

Figure 6 Teratogenicity of nilotinib. (A) Foetus number per mother, (B) foetus weight/placenta weight ratio, (C) morphological assessment of foetuses, and (D) bodyweight of the pups.
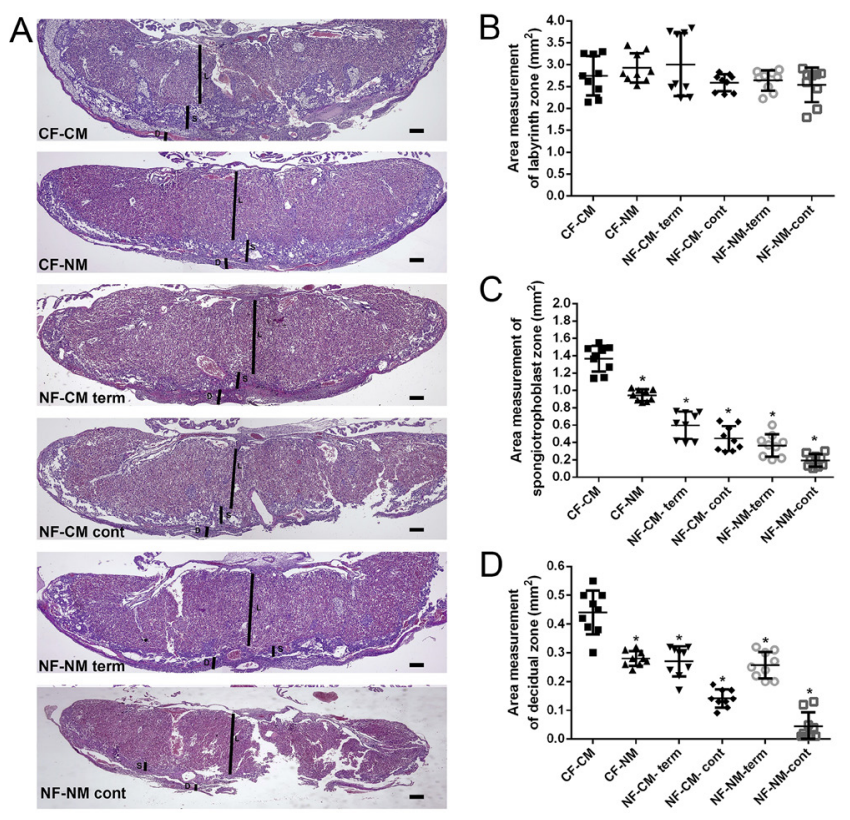

Figure 7 (A) Whole placenta sections (at the site of umbilical cord level for normalising the size of the placenta in all groups) stained with haematoxylin-eosin (L, Labyrinth zone; S, Spongiotrophoblast zone; D, Decidua) and (B) areal measurements and comparisons of the labyrinth zone, (C) spongiotrophoblast zone and (D) decidua among experimental groups. 


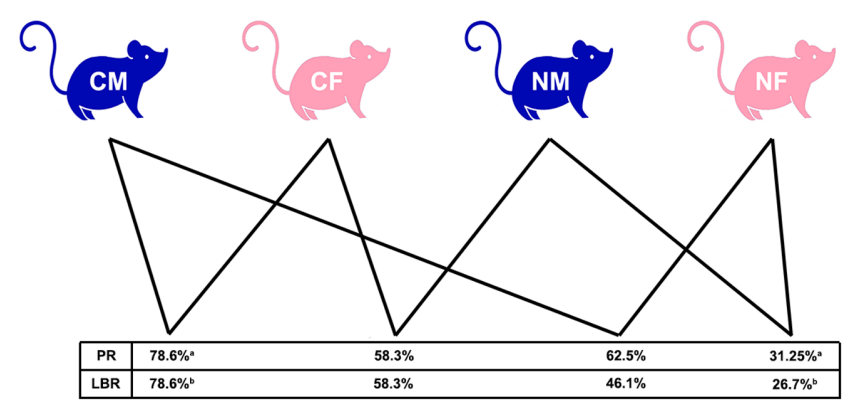

Figure 8 Pregnancy (PR) and live birth rates (LBR) of the parent mice who were involved in the experimental and the control groups. Similar superscript letters indicate significant difference between groups, (A), $P=0.012$, (B), $P=0.007$. CM, control male; $\mathrm{CF}$, control female; NM, nilotinib received male; $N F$, nilotinib received female.

occurred (58.3\%), in CMNF group was calculated in 16 females and 10 pregnancies occurred (62.5\%), in NMNF group was calculated in 16 females and 5 pregnancies occurred $(31.25 \%)$. Live birth rate in CMCF group was calculated in 14 females and 11 offspring were born $(78.6 \%)$, in NMCF group was calculated in 12 females and 7 offspring were born $(58.3 \%)$, in CMNF group was calculated in 13 females and 6 offspring were born $(46.1 \%)$, in NMNF group was calculated in 15 females and 4 offspring were born (26.7\%). Significant difference was evident only in between CMCF and NMNF groups both in pregnancy rates $(P=0.012)$ and live birth rates $(P=0.007)$.

When all naturally born offspring were matched with a control mouse of the opposite sex, it was understood that the number of pups in each group was not sufficient to be evaluated by any statistical method. Even though there is a tendency of change between groups, it was not possible to express this in terms of significance, so the fertility rates of the offspring appeared as in Table 1, depicted according to their specific genders and in total.

\section{Discussion}

The findings revealed in this study showed that nilotinib is gonadotoxic and the adverse reproductive effects of nilotinib occurred both acutely and in long term despite drug cessation. As TKIs have been reported to have teratogenic effects in natural pregnancy cases, there is a consensus that patients planning pregnancy or have conceived need to stop the treatment to avoid potential teratogenic side effects. However, this level of knowledge relies mostly on expert opinions and single case presentations. If pregnancy is planned or realised incidentally, the timing of discontinuation of the drug is of great importance for the foetus and the expectant mother (Dou et al. 2019).

Considering the joint contribution of both sexes in pregnancy formation, concerns might arise about the loss of reproductive functions in men, who use TKI, or the health of the foetus derived from these men. However, reports that approach this issue are rare in the literature. Reproductive restriction and contraception are not recommended for the male partner who uses TKI. However, women should use contraceptive methods during the use of TKI because of the teratogenic effect of drugs (Abruzzese et al. 2016). Although TKIs are used to suppress the increased tyrosine kinase activity caused by $\mathrm{BCL}-\mathrm{ABL}$ fusion, many non-target tyrosine kinases can also be inhibited (Kierszenbaum 2006, Nurmio et al. 2007, Mcginnis et al. 2011, Wan et al. 2014). Although there are case reports of healthy birth with natural conception with men on nilotinib (Aota et al. 2020), there are no clinical or animal studies with long-term follow-up of children born in these circumstances. In pregnancies caused by men, who were using imatinib due to $\mathrm{CML}$, approximately $85 \%$ of the reports resulted in a healthy live birth. In comparison, 3\% resulted in induced abortion, and $2 \%$ in spontaneous abortion. In a few of these pregnancies, intrauterine death, mild small intestine rotation, hydrocephalus, and congenital malformation were reported. In 81 pregnancies caused by men on dasatinib treatment, spontaneous abortion occurred in two cases, while syndactyly was observed in one. Although syndactyly rates are much rarer in the average population (almost 1 in 2500), it is not possible to link it with the drug from a case-cohort study. Five normal live births were reported in five pregnancies, where the male partner used nilotinib (Abruzzese et al. 2016). Carlier et al. compiled the results of pregnancies caused by men using TKI in the French health system. The results of 11 births arising from 14 pregnancies caused by men using imatinib, dasatinib, and nilotinib, were evaluated where one of the pregnancies resulted

Table 1 Male and female mice (columns 2 and 4) retrieved from the experimental groups were matched with a control opposite gender mouse, and their fertility was calculated according to the pregnancy rate they achieved.

\begin{tabular}{|c|c|c|c|c|c|c|}
\hline $\begin{array}{l}\text { Born in the } \\
\text { experiment group }\end{array}$ & Males $(n)$ & $\begin{array}{c}\text { Male offspring } \\
\text { achieved pregnancy, } \\
\boldsymbol{n}(\%)\end{array}$ & Females $(n)$ & $\begin{array}{c}\text { Female offspring } \\
\text { achieved pregnancy, } \\
\boldsymbol{n}(\%)\end{array}$ & Total $(n)$ & $\begin{array}{c}\text { Total offspring } \\
\text { achieved pregnancy, } \\
n(\%)\end{array}$ \\
\hline F-CMCF & 3 & $3(100 \%)$ & 3 & $3(100 \%)$ & 6 & $6(100 \%)$ \\
\hline F-NMCF & 3 & $3(100 \%)$ & 2 & $2(100 \%)$ & 5 & $5(100 \%)$ \\
\hline F-CMNF-Term & 3 & $2(66.6 \%)$ & 3 & $1(33.3 \%)$ & 6 & $3(50 \%)$ \\
\hline F-CMNF-Cont & 3 & $2(66.6 \%)$ & 3 & $0(0 \%)$ & 6 & $2(33.3 \%)$ \\
\hline F-NMNF-Term & 2 & $2(100 \%)$ & 3 & $2(66.6 \%)$ & 5 & $4(83.3 \%)$ \\
\hline F-NMNF-Cont & 1 & $0(0 \%)$ & 2 & $0(0 \%)$ & 3 & $0(0 \%)$ \\
\hline
\end{tabular}


with spontaneous abortion and two of them with induced abortion; anomaly was detected in three babies (Carlier et al. 2017). All this data reveals that there is insufficient evidence regarding whether spermatozoon freezing has to be recommended to patients for fertility preservation before using TKIs.

It was previously reported that nilotinib causes toxicities in somatic organs, such as the skin, lungs, and heart (Atallah 2011, Go et al. 2013, Moslehi \& Deininger 2015, Ransohoff \& Kwong 2017). However, in this study, no significant weight change was detected in the major organs in the female and male experimental animals compared to the control group. Unlike these results, testicular weights per body weight after the use of nilotinib in male mice were found significantly reduced. These findings indicate a deterioration in the testicular structure. Our findings clearly show that testicular toxicity occurs with the nilotinib use in the animal model. Testicular weights, together with the morphologic Johnsen's score and proliferation rates, decreased with the use of nilotinib, and apoptosis increased. The deterioration in the seminiferous tubules' general structure was detected, and the spermatogenic cells' displacement on the tubule wall was observed. Some of these findings persisted in the post-nilotinib group, except in the testis weight/body weight ratio, the significant difference disappeared. However, impairments in testicular apoptosis, proliferation, and testis histology continued. In this sense, it is thought that the increased deterioration in spermatogenesis and testicular apoptosis continues for a long time even if the drug is terminated. When the pharmacological brochure of nilotinib was examined, it was emphasised that it did not affect sperm parameters (Abruzzese et al. 2014). Oppositely, in our results, the male mice receiving nilotinib caused a lower pregnancy rate $(58.3 \%$ vs $78.5 \%$ compared to the control) and their testicular histological scores showing spermatogenesis were lower.

Considering the data from female mice, it was found that there was no significant difference in the ovary weight per bodyweight ratio after the use of nilotinib in mice. However, a significant decrease occurred in the primordial follicles that constitute the ovarian reserve. It was demonstrated that proliferation, which is an essential marker for follicle development, decreased in this group compared to the control, and apoptosis increased. After discontinuation of nilotinib, mice were followed for 15 days without medication, and it was evaluated whether the gonadotoxicity was reversible. When they were compared to the same age controls, the number of primordial follicles was found to be decreased insignificantly. Nevertheless, these women are still candidates for fertility preservation procedures as the markers of apoptosis and proliferation did not get better after several ovarian cycles in mice.

Another issue that has no definitive answer in the literature is how to approach pregnancies occurring during nilotinib use. Although there is an accumulation of knowledge about first-generation TKIs, the more potent and widespread use of the next-generation drugs brings concerns. However, the data on the subject appears mostly in the form of case reports. In a case report published by Conchon et al. (2009), a woman was presented who was diagnosed with CML during her first pregnancy but gave birth to a healthy offspring before starting the treatment. After 2 years, she conceived unplanned while under nilotinib treatment, continued in the normal course of pregnancy, and reached normal birth with the discontinuation of nilotinib treatment at the seventh week (Conchon et al. 2009). In a case report of another patient, who became pregnant while under dasatinib treatment, a dramatic foetal loss occurred despite the discontinuation of the drug at the seventh week, and the presence of dasatinib was detected in the placenta and amniotic fluid (Berveiller et al. 2012). In 2013, Zhou et al. reported a patient series about pregnancies in 18 women and 7 men on TKIs, with their drug-free partners. Only one pregnancy from a male TKI-user resulted in premature birth, and the other six ended without complications. Eight elective and three spontaneous abortions were noted in women in whom pregnancy occurred during drug use. TKI usage duration in cases that ended with abortion was over 6 weeks (Zhou et al. 2013). In our study, no significant differences were observed between the groups in terms of the number of foetuses where nilotinib was ceased or continued upon pregnancy.

When any of the parents received nilotinib, the foetus had slightly smaller placentas than the groups that did not, and the abnormalities were mainly limited to the spongiotrophoblast and decidual zones. Significant reduction in the spongiotrophoblast zone after maternal and/or paternal nilotinib treatment may indicate that both hormonal and glycogen metabolism may be impaired, and this information deserves further investigation (Woods et al. 2018). The effect of nilotinib on decidualisation is unknown in the literature. It has been found that imatinib treatment reduces endometrial stromal cell proliferation and invasion (Griffith et al. 2010, Wang et al. 2015). Therefore, nilotinib may have adverse effects on endometrial physiology and thus on placental development. A recent study found that long-term imatinib exposure has a long-term impact on placentation with significant changes in epigenetic markers of imprinted genes in the mouse placenta (Salem et al. 2019). Altogether, it can be suggested that a long wash-out period before pregnancy or extra monitoring for possible placental insufficiency may be advisable for patients under TKI treatment in the clinic.

Our results indicate that the use of nilotinib reduces the likelihood of achieving pregnancy in both males and females compared to controls. This effect increases significantly if the nilotinib was used in both genders. In our study, the first-generation offspring fertility 
was also evaluated. In fact, the numbers of offspring for this experiment group could not be predicted at the beginning of the study as it depended on the pregnancy numbers in previous matched groups. As the numbers of offspring included in this experiment appeared insufficient to create a statistical $P$-value, the outcomes were presented as percentage values, which is a limitation for the current study. The numbers of offspring included in this experiment in both genders were depicted in Table 1. New experimental and clinical follow-up studies are certainly needed to investigate the effect of nilotinib on fertility of offspring in more detail. Results revealed that offspring with any or both parents, when received nilotinib, might have problems in obtaining pregnancy in their adulthood when matched with control animals. It is possible to hypothesis that epigenetic changes or alterations in gonad development may be affecting fertility in adulthood.

In conclusion, this is the first study to examine the gonadotoxic and teratogenic effects of nilotinib in the female and male reproductive systems, the fertility of the first-generation offspring, and whether these effects are reversible, using an animal model. The results of our study are of great importance in terms of clinical use of nilotinib, because patients starting the drug should be informed about fertility protection options, and pregnancy tests should be performed. The main reason for offering gamete or gonad cryopreservation is in nilotinib's potential long-lasting effects on gonads. With a high chance of cure, CML patients' longterm life quality receiving nilotinib should be taken into account, considering their fertility. Although the data presented in this study have been demonstrated in experimental animals, it will provide crucial information on the approach to CML patients in the clinic.

\section{Declaration of interest}

The authors declare that there is no conflict of interest that could be perceived as prejudicing the impartiality of the research reported.

\section{Funding}

This work was supported by the Scientific and Technological Research Council of Turkey-TUBITAK (grant number: 118S068).

\section{Author contribution statement}

N K, D A, and C C O performed the experiments; S O and C C $\mathrm{O}$ analysed the results, made the figures and wrote the paper; S O, G C S and C C O designed the research.

\section{Acknowledgements}

Presented partially as a poster presentation in abstract form and received $\mathrm{ASH}$ Abstract Achievement Award at the 56th annual meeting of the American Society of Hematology, San Francisco, CA, December 2014.

\section{References}

Abruzzese E, Trawinska MM, Perrotti AP \& De Fabritiis P 2014 Tyrosine kinase inhibitors and pregnancy. Mediterranean Journal of Hematology and Infectious Diseases 6 e2014028. (https://doi.org/10.4084/ MJHID.2014.028)

Abruzzese E, Trawinska MM, De Fabritiis P \& Baccarani M 2016 Management of pregnant chronic myeloid leukemia patients. Expert Review of Hematology 9 781-791. (https://doi.org/10.1080/17474086 .2016.1205479)

Alizadeh H, Jaafar H, Rajnics P, Khan MI \& Kajtar B 2015 Outcome of pregnancy in chronic myeloid leukaemia patients treated with tyrosine kinase inhibitors: short report from a single centre. Leukemia Research 39 47-51. (https://doi.org/10.1016/j.leukres.2014.10.002)

Aota Y, Udagawa S, Honda T, Okuda Y \& Gotoh A 2020 Delivery of a healthy newborn by the partner of a patient with $\mathrm{cml}$ undergoing treatment with nilotinib. Gan To Kagaku Ryoho: Cancer and Chemotherapy 47 811-813.

Atallah E 2011 Nilotinib cardiac toxicity: should we still be concerned? Leukemia Research $35 \quad 577-578 . \quad$ (https://doi.org/10.1016/j. leukres.2011.01.021)

Barkoulas T \& Hall PD 2018 Experience with dasatinib and nilotinib use in pregnancy. Journal of Oncology Pharmacy Practice 24 121-128. (https:// doi.org/10.1177/1078155217692399)

Berveiller P, Andreoli A, Mir O, Anselem O, Delezoide AL, Sauvageon H, Chapuis N \& Tsatsaris V 2012 A dramatic fetal outcome following transplacental transfer of dasatinib. Anti-Cancer Drugs 23 754-757. (https://doi.org/10.1097/CAD.0b013e328352a8fe)

Carlier P, Markarian M, Bernard N, Lagarce L, Dautriche A, Bene J, Fouilhe Sam-Lai N \& Eftekhari P 2017 Pregnancy outcome among partners of male patients receiving imatinib, dasatinib or nilotinib in chronic myeloid leukemia: reports collected by the French network pharmacovigilance centers. Archives of Gynecology and Obstetrics 295 269-271. (https://doi.org/10.1007/s00404-016-4262-z)

Chen Y, Wang H, Qi N, Wu H, Xiong W, Ma J, Lu Q \& Han D 2009 Functions of tam rtks in regulating spermatogenesis and male fertility in mice. Reproduction 138 655-666. (https://doi.org/10.1530/REP-09-0101)

Cheon J, Ahn JW, Park KM, Lee G \& Jo YS 2016 Teratogenic effect of radotinib: case report. Anticancer Research 36 6599-6601. (https://doi. org/10.21873/anticanres.11265)

Conchon M, Sanabani SS, Bendit I, Santos FM, Serpa M \& DorliacLlacer PE 2009 Two successful pregnancies in a woman with chronic myeloid leukemia exposed to nilotinib during the first trimester of her second pregnancy: case study. Journal of Hematology and Oncology 2 42. (https://doi.org/10.1186/1756-8722-2-42)

Dou X, Qin Y, Huang X \& Jiang Q 2019 Planned pregnancy in female patients with chronic myeloid leukemia receiving tyrosine kinase inhibitor therapy. Oncologist 24 e1141-e1147. (https://doi.org/10.1634/ theoncologist.2019-0109)

Go SI, Lee WS, Lee GW, Kang JH, Kang MH, Lee JH \& Kim HG 2013 Nilotinib-induced interstitial lung disease. International Journal of Hematology 98 361-365. (https://doi.org/10.1007/s12185-013-1398-5)

Griffith JS, Binkley PA, Kirma NB, Schenken RS, Witz CA \& Tekmal RR 2010 Imatinib decreases endometrial stromal cell transmesothial migration and proliferation in the extracellular matrix of modeled peritoneum. Fertility and Sterility 94 2531-2535. (https://doi.org/10.1016/j. fertnstert.2010.04.018)

Ijiri TW, Mahbub Hasan AK \& Sato K 2012 Protein-tyrosine kinase signaling in the biological functions associated with sperm. Journal of Signal Transduction 2012 181560. (https://doi.org/10.1155/2012/181560)

Jensen MR, Brüggen J, Dilea C, Mestan J \& Manley PW 2006 AMN107: Efficacy of the Selective Bcr-Abl Tyrosine Kinase Inhibitor in a Murine Model of Chronic Myelogenous Leukemia. American Association for Cancer Research. 
Johnsen SG 1970 Testicular biopsy score count - a method for registration of spermatogenesis in human testes: normal values and results in 335 hypogonadal males. Hormones 1 2-25. (https://doi. org/10.1159/000178170)

Kantarjian HM, O'Brien S, Cortes J, Giles FJ, Rios MB, Shan J, Faderl S, Garcia-Manero G, Ferrajoli A, Verstovsek S et al. 2003 Imatinib mesylate therapy improves survival in patients with newly diagnosed philadelphia chromosome-positive chronic myelogenous leukemia in the chronic phase: comparison with historic data. Cancer 98 2636-2642. (https:// doi.org/10.1002/cncr.11831)

Kierszenbaum AL 2006 Tyrosine protein kinases and spermatogenesis: truncation matters. Molecular Reproduction and Development 73 399-403. (https://doi.org/10.1002/mrd.20456)

Mcginnis LK, Carroll DJ \& Kinsey WH 2011 Protein tyrosine kinase signaling during oocyte maturation and fertilization. Molecular Reproduction and Development 78 831-845. (https://doi.org/10.1002/mrd.21326)

Moslehi JJ \& Deininger M 2015 Tyrosine kinase inhibitor-associated cardiovascular toxicity in chronic myeloid leukemia. Journal of Clinical Oncology 33 4210-4218. (https://doi.org/10.1200/JCO.2015.62.4718)

Nurmio M, Toppari J, Zaman F, Andersson AM, Paranko J, Soder O \& Jahnukainen K 2007 Inhibition of tyrosine kinases pdgfr and c-kit by imatinib mesylate interferes with postnatal testicular development in the rat. International Journal of Andrology 30 366-376; discussion 376. (https://doi.org/10.1111/j.1365-2605.2007.00755.x)

Palani R, Milojkovic D \& Apperley JF 2015 Managing pregnancy in chronic myeloid leukaemia. Annals of Hematology 94 S167-S176. (https://doi. org/10.1007/s00277-015-2317-z)

Ransohoff JD \& Kwong BY 2017 Cutaneous adverse events of targeted therapies for hematolymphoid malignancies. Clin Lymphoma Myeloma Leuk 17 834-851. (https://doi.org/10.1016/j.clml.2017.07.005)

Ross MH \& Pawlina W 2016 Histology: A Text and Atlas. Philadelphia: Wolters Kluver Health.

Rosti G, Palandri F, Castagnetti F, Breccia M, Levato L, Gugliotta G, Capucci A, Cedrone M, Fava C, Fava C, Intermesoli T et al. 2009 Nilotinib for the frontline treatment of $\mathrm{ph}(+)$ chronic myeloid leukemia. Blood 114 4933-4938. (https://doi.org/10.1182/blood-2009-07-232595)
Salem W, Li K, Krapp C, Ingles SA, Bartolomei MS, Chung K, Paulson RJ, Nowak RA \& Mcginnis LK 2019 Imatinib treatments have long-term impact on placentation and embryo survival. Scientific Reports 92535. (https://doi.org/10.1038/s41598-019-39134-0)

US Food and Drug Administration 2018 Fda approves nilotinib for pediatric patients with newly diagnosed or resistant/intolerant $\mathrm{ph}+\mathrm{cml}$ in chronic phase [Online]. US Food and Drug Administration. (available at: https://www.fda.gov/drugs/resources-information-approved-drugs/ fda-approves-nilotinib-pediatric-patients-newly-diagnosed-orresistantintolerant-ph-cml-chronic). Accessed on 12 November 2.

Wan HT, Mruk DD, Tang EI, Xiao X, Cheng YH, Wong EW, Wong CK \& Cheng CY 2014 Role of non-receptor protein tyrosine kinases in spermatid transport during spermatogenesis. Seminars in Cell and Developmental Biology 30 65-74. (https://doi.org/10.1016/j.semcdb.2014.04.013)

Wang XR, Wang CD, Liu XM, Bao HC, Qu QL \& Hao CF 2015 Effect of pdgf-rb antagonist imatinib on endometrial injury repairing in mouse model. Asian Pacific Journal of Tropical Medicine 8 555-559. (https:// doi.org/10.1016/j.apjtm.2015.06.001)

Woods L, Perez-Garcia V \& Hemberger M 2018 Regulation of placental development and its impact on fetal growth - new insights from mouse models. Frontiers in Endocrinology 9 570. (https://doi.org/10.3389/ fendo.2018.00570)

Zhou L, You JH, Wu W, Li JM, Shen ZX \& Wang AH 2013 Pregnancies in patients with chronic myeloid leukemia treated with tyrosine kinase inhibitor. Leukemia Research 37 1216-1221. (https://doi.org/10.1016/j. leukres.2013.07.020)

Received 6 October 2020

First decision 29 October 2020

Revised manuscript received 2 January 2021

Accepted 11 January 2021 